$$
\text { phys. stat. sol. (b) 50, } 187 \text { (1972) }
$$

Subject classification: 10.2 and $20.3 ; 19 ; 22.5 .2$

Institut de Physique, Université de Neuchâtel, Switzerland

\title{
Weak Magnetic Field Enhancement of the Luminescence from F Centre Pairs in Alkali Halides
}

\author{
By \\ C. Jaccard, Y. Ruedin, M. Aggerter, and P.-A. SchnegG
}

\begin{abstract}
Whenever a member of a $\mathbf{F}$ centre pair is excited optically at low temperature, it can return to the ground state either radiatively or by formation of a $\mathrm{F}^{\prime}$ centre, the excited electron being transferred by a fast tunnel effect to the neighbouring centre. This process is dependent on the spin symmetry of the pair, which is in turn determined by the competitive influence of the different local hyperfine fields and of an applied magnetic field. The latter causes a decrease of the tunnelling probability, i.e. an increase of the luminescent yield in two steps; the first critical value is given by the hyperfine field and the second one by the temperature according to the Boltzmann factor. The quantum yield is found to have an exponential dependence of the concentration, of the volume in which tunnelling is possible, and of the average tunnelling frequency. Spin-lattice relaxation and EPR, by mixing between the spin state populations, reduce the effect. Application of the theory to experimental values obtained for the luminescence intensity as a function of the applied field in $\mathrm{KI}, \mathrm{KCl}$, and $\mathrm{KBr}$ yields the average tunnelling frequency (about ten times the radiative probability), the effective range ( $85 \AA$ ), and the absolute value of the luminescent quantum yield.
\end{abstract}

Lorsqu'un membre d'une paire de centres $\mathrm{F}$ est excité optiquement à basse température, il peut retourner à l'état fondamental soit radiativement, soit en formant un centre $\mathrm{F}^{\prime}$, l'électron excité étant transféré par un effet tunnel rapide au centre voisin. Ce processus est dépendant de la symétrie de spin de la paire qui est à son tour déterminée par l'influence concurrentielle des différents champs hyperfins et d'un champ magnétique appliqué. Ce dernier cause une diminution de la probabilité de tunnel, c'est-à-dire une augmentation du rendement luminescent en deux étapes, la première valeur critique étant donnée par le champ hyperfin et la seconde par la température (selon le facteur de Boltzmann). On trouve que le rendement quantique est une fonction exponentielle de la concentration, $d u$ volume dans lequel l'effet tunnel est possible, ainsi que de la fréquence moyenne de transfert. La relaxation spin-réseau et la RPE, en redistribuant les spins entre leurs divers états, réduisent cet effet. Cette théorie est appliquée à des mesures faites dans $\mathrm{KJ}$, $\mathrm{KCl}$ et $\mathrm{KBr}$ de l'intensité de la luminescence en fonction du champ magnétique. On en tire la fréquence de tunnel moyenne (environ dix fois la probabilité radiative), la distance efficace $(85 \AA)$ et la valeur absolue du rendement quantique luminescent.

\section{Introduction}

F centres are among the simplest defects in ionic crystals: an electron located in an anion vacancy. Although they have been discovered fourty years ago and studied quite intensively in the last two decades, the phenomena following a photon absorption still present interesting problems. F centres spend only about $10^{-12} \mathrm{~s}$ in the excited state $\mathrm{F}^{*}$. The lattice responds to the change of the electron distribution by a local relaxation, dissipating part of the energy and leading to a relaxed excited state $\tilde{\mathrm{F}}^{*}$. At low temperature, an isolated centre 
remains in this state for a relatively long time (between 1 and $0.1 \mu \mathrm{s}$ in most of alkali halides) before it emits a photon and returns to the ground state [1]. In this case, the luminescent quantum yield, defined as the ratio "number of emitted photons to number absorbed of photons", is unity. At higher temperature (above $100^{\circ} \mathrm{K}$ ), the electron has the possibility to be thermally activated into the conduction band, from which it decays to the ground state by nonradiative processes. This produces a decrease of both the quantum yield and the average lifetime, as it has been observed by Bosi et al. [2]. If the F centre concentration is high enough, there is a finite probability that two centres form a pair within a certain critical distance. Markham et al. [3] suggested for this case a third disexcitation path: the electron of the excited centre is transferred by tunnel effect to its neighbour, thereby forming a $F^{\prime}$ centre and an anion vacancy, and the pair returns then to its ground state $\mathbf{F}^{0}+\mathbf{F}^{0}$ by some nonradiative mechanism. The existence of this process has been demonstrated by Porret and Lüty [4]: at $1.7{ }^{\circ} \mathrm{K}$ a magnetic field of $80 \mathrm{kG}$ polarizes the spins completely. Since the two electrons of the $\mathrm{F}^{\prime}$ centre in its ground state must have antiparallel spins, the transfer is no longer possible. This produces an increase of the radiative quantum yield because the radiative process is the only way left for the disexcitation. Such a pair effect confers also to the luminescent yield a strong dependence of the $\mathbf{F}$ concentration, as it has been discovered by Miehlich [6]. This mechanism is also supported by experiments performed in our laboratory by Ruedin and co-workers [5] who detected optically the paramagnetic resonance of the $\mathrm{F}$ centre in its ground and excited state.

A closer investigation of the variation of the luminescence intensity induced by a magnetic field has revealed a first stage of this effect, occurring at low fields of the order of the measured EPR linewidth. Typically a quantum yield increase of some ten percent is observed in crystals having $10^{17} \mathrm{~F}$ centres per $\mathrm{cm}^{3}$. This can be explained by a competing influence on the pairs of the applied field and of the local hyperfine fields, which changes the character of the wave functions and therefore strongly modify the tunnel probability.

This paper attempts to calculate the spin state of a two-electron pair $\widetilde{\mathrm{F}}^{*}+\mathbf{F}$ and its implication for tunnelling by taking into account the centre concentration. The effects of the spin-lattice relaxation and of a microwave field are considered, as well as effects of second order such as the centre correlation and the exciting light intensity. In the last section, experiments performed on $\mathrm{KCl}$, $\mathrm{KBr}$, and $\mathrm{KI}$ are compared with the theory and the tunnelling parameters are determined.

\section{Pair States and Tunnelling}

We consider first a $\widetilde{\mathbf{F}} *+\mathbf{F}$ pair at liquid helium temperature. In this range, the Boltzmann factor for the nuclear spins is very near unity, so that the nuclear orientations can be assumed to be distributed at random. Moreover, the nuclear spin-lattice relaxation time is certainly longer than the characteristic times we are dealing with, i.e. $1 \mu$ s, so that the nuclear field is quasi-stationary. We assume also that the interval between the pair members is small enough to ensure a finite tunnelling probability, but large enough to make the two local nuclear fields $\boldsymbol{H}_{\mathrm{N}}$ and $\boldsymbol{H}_{\mathrm{N}}^{*}$ different. If $\boldsymbol{H}_{0}$ is the applied field, the centres are subjected to $\boldsymbol{H}=\boldsymbol{H}_{\mathbf{0}}+\boldsymbol{H}_{\mathrm{N}}$ and $\boldsymbol{H}^{*}=\boldsymbol{H}_{0}+\boldsymbol{H}_{\mathrm{N}}^{*}=\boldsymbol{H}+\boldsymbol{H}^{\prime}$, with the field difference $\boldsymbol{H}^{\prime}=\boldsymbol{H}_{\mathrm{N}}^{*}-\boldsymbol{H}_{\mathrm{N}}$ (Fig. 1). 
lig. 1. Magnetic fields acting on $F$ centres : $\boldsymbol{H}_{0}$ is the applied field, $\boldsymbol{H}_{\mathrm{N}}$ and $\boldsymbol{H}_{\mathrm{N}}^{*}$ are the local hyperfine fields

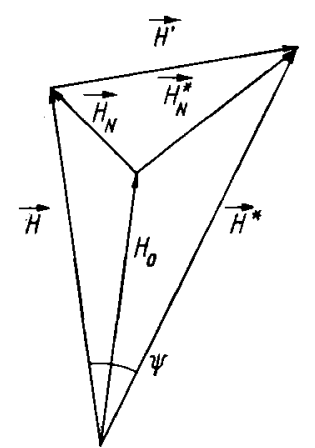

At zero local field $\boldsymbol{H}=\boldsymbol{H}^{*}=0$, the two-electron system of the pair can be described by a symmetric and an antisymmetric combination of the singlecentre orbital wave functions $\varphi$ and $\varphi^{*}$ :

$$
\Phi_{\mathrm{s}, \mathrm{a}}=\frac{1}{\sqrt{2}}\left[\varphi^{*}(1) \varphi(2) \pm \varphi(1) \varphi^{*}(2)\right]
$$

to be multiplied by spin functions of the opposite symmetry

$$
\begin{aligned}
\sigma_{ \pm 1} & =| \pm \pm\rangle, \\
\sigma_{0} & =\frac{1}{\sqrt{2}}(|+-\rangle+|-+\rangle), \quad \alpha=\frac{1}{\sqrt{2}}(|+-\rangle-|-+\rangle) .
\end{aligned}
$$

The products should be eigenfunctions of an orbital Hamiltonian in which the spin-orbit coupling is accounted for.

The interaction between the spins and the magnetic fields can be described by the following Hamiltonian:

$$
\mathscr{H}_{\mathrm{m}}=g^{*} \beta \boldsymbol{H}^{*} \cdot \boldsymbol{S}_{i}+g \beta \boldsymbol{H} \cdot \boldsymbol{S}_{j} .
$$

Since the fields are space-dependent, $\mathbf{S}_{i}$ should act on the first electron for the part of the wave function containing $\varphi^{*}(1)$ and $S_{j}$ for the part of the wave function containing $\varphi(1)$. A corresponding condition applies to the second electron.

The exact diagonalization of the Hamiltonian operator yields the four eigenstates, in which the angle $\psi$ between $\boldsymbol{H}$ and $\boldsymbol{H}^{*}$ governs the amount of mixing:

$$
\begin{aligned}
& \Psi_{1}=\frac{1}{\sqrt{2}} \sin \left(\frac{\psi}{2}\right)\left(\Phi_{\mathrm{s}} \alpha+\Phi_{\mathrm{a}} \sigma_{0}\right)+\cos \left(\frac{\psi}{2}\right) \Phi_{\mathrm{a}} \sigma_{+1} \\
& \Psi_{2}=\frac{1}{\sqrt{2}} \cos \left(\frac{\psi}{2}\right)\left(\Phi_{\mathrm{s}} \alpha+\Phi_{\mathrm{a}} \sigma_{0}\right)-\sin \left(\frac{\psi}{2}\right) \Phi_{\mathrm{a}} \sigma_{+1} \\
& \Psi_{3}=\frac{1}{\sqrt{2}} \cos \left(\frac{\psi}{2}\right)\left(\Phi_{\mathrm{s}} \alpha-\Phi_{\mathrm{a}} \sigma_{0}\right)-\sin \left(\frac{\psi}{2}\right) \Phi_{\mathrm{a}} \sigma_{-1} \\
& \Psi_{4}=\frac{1}{\sqrt{2}} \sin \left(\frac{\psi}{2}\right)\left(\Phi_{\mathrm{s}} \alpha-\Phi_{\mathrm{a}} \sigma_{0}\right)+\cos \left(\frac{\psi}{2}\right) \Phi_{\mathrm{a}} \sigma_{-1}
\end{aligned}
$$


The corresponding energies are

$$
\varepsilon_{1,4}= \pm\left(\frac{1}{2} g^{*} \beta H^{*}+\frac{1}{2} g \beta H\right), \quad \varepsilon_{2,3}= \pm\left(\frac{1}{2} g^{*} \beta H^{*}-\frac{1}{2} g \beta H\right) .
$$

This procedure is correct if $\varphi$ and $\varphi^{*}$ are orthogonal. But since we deal with a tunnel effect, we have to assume some overlap, and therefore to consider the system above as a first approximation.

For magnetic fields smaller than $5 \mathrm{kG}$ (at LHeT), the occupation probability for each of the four states is equal to $1 / 4$. At higher fields, the Boltzmann factor has to be considered. However, as long as the spin-lattice relaxation time is longer than the radiative lifetime [5], an electron in the relaxed excited state has no time to thermalize, so that the occupation probabilities are determined by those of the ground state. The occupation probabilities are then

if we assume

$$
\begin{aligned}
& v_{1}+v_{4}=\frac{\cosh \left(g \beta H_{0} / k T\right)}{2 \cosh ^{2}\left(g \beta H_{0} / 2 k T\right)}, \\
& v_{2}+\nu_{3}=\frac{1}{2 \cosh ^{2}\left(g \beta H_{0} / 2 k T\right)}
\end{aligned}
$$

$$
\left|\boldsymbol{H}_{0}\right| \gg\left|\boldsymbol{H}_{\mathrm{N}}\right| \text { and }\left|\boldsymbol{H}_{\mathrm{N}}^{*}\right|
$$

In the final state of the tunnelling process, both electrons form a $F^{\prime}$ centre and have the same orbital wave function but an antisymmetric spin function. For each of the initial states the tunnelling frequency has therefore the following form:

$$
\omega_{\mathrm{t} 1}=\omega_{\mathrm{t} 4}=\omega_{\mathrm{t} 0}(R) \frac{1}{\sqrt{2}} \sin \left(\frac{\psi}{2}\right), \quad \omega_{\mathrm{t} 2}=\omega_{\mathrm{t} 3}=\omega_{\mathrm{t} 0}(R) \frac{1}{\sqrt{2}} \cos \left(\frac{\psi}{2}\right) .
$$

The function $\omega_{\mathrm{t} 0}(R)$ depends only upon the orbital parts of the wave functions and varies strongly with the pair interval $R[7,8]$.

If we define $W(R)=\omega_{\mathrm{t} 0}(R) / w_{\mathrm{r}}$ by taking the radiative transition probability for the $\widetilde{\mathrm{F}}^{*}$ centre $w_{\mathrm{r}}$ as a unit, the tunnelling probability for one pair becomes at low field $\left(H_{0}<5 \mathrm{kG}\right)$

$$
P_{\mathrm{t}}=P_{1 \mathrm{t}}\left(R, H_{0}\right)=\frac{1}{2}\left[\frac{(W / \sqrt{2}) \sin (\psi / 2)}{1+(W / \sqrt{2}) \sin (\psi / 2)}+\frac{(W / \sqrt{2}) \cos (\psi / 2)}{1+(W / \sqrt{2}) \cos (\psi / 2)}\right]
$$

and at high field $\left(H_{0}>5 \mathrm{kG}\right)$

$$
P_{\mathrm{t}}=P_{2 \mathrm{t}}\left(R, H_{0} / T\right)=\frac{1}{2} \frac{W / \sqrt{2}}{1+(W / \sqrt{2})}\left[\cosh \left(\frac{g \beta H_{0}}{2 k T}\right)\right]^{-2} .
$$

The number of pairs can be evaluated from the centre concentration $n$ if one assumes a random spatial distribution of the centres. A single excited centre can have several neighbours within a tunnelling distance and this can be accounted for by the following consideration. Each excited centre is placed at the origin of a coordinate system, and the volume around it is divided in small elements $\Delta V$, numbered with an index $j$. The probability of having $0,1,2, \ldots$ 
neighbours in such volumes is then given by Poisson's law:

$$
\begin{aligned}
& p(0)=\exp (-n \Delta V) \approx 1-n \Delta V, \\
& p(1)=n \Delta V \exp (-n \Delta V) \approx n \Delta V, \\
& p(2) \approx p(3) \approx \cdots \approx 0 .
\end{aligned}
$$

The luminescence probability is given by

$$
P_{\mathrm{L}}=p(0) 1+p(1)\left(1-P_{\mathrm{t}}\right)=1-n \Delta V P_{\mathrm{t}} .
$$

The radiative quantum yield of each $\widetilde{\mathrm{F}}^{*}$ centre is just the probability that its electron does not tunnel toward any neighbour situated in any volume element:

$$
\begin{aligned}
& \eta=\prod_{j}\left[1-n \Delta V P_{\mathrm{t}}\left(\boldsymbol{r}_{j}\right)\right] \approx 1-n \sum_{j} \Delta V P_{\mathrm{t}}\left(\boldsymbol{r}_{j}\right)+ \\
& +\frac{1}{2} n^{2} \sum_{j} \sum_{k}(\Delta V)^{2} P_{\mathrm{t}}\left(\boldsymbol{r}_{j}\right) P_{\mathrm{t}}\left(\boldsymbol{r}_{k}\right)-\frac{1}{6} n^{3} \sum_{j} \sum_{k} \sum_{l}(\Delta V)^{3} P_{\mathrm{t}}\left(\boldsymbol{r}_{j}\right) P_{\mathrm{t}}\left(\boldsymbol{r}_{k}\right) P_{\mathrm{t}}\left(\boldsymbol{r}_{l}\right)+\cdots
\end{aligned}
$$

The total luminescent quantum yield is the average of this expression for all combinations of $P_{t}\left(r_{1}\right)$, i.e. for all the combinations of the nuclear fields $\boldsymbol{H}_{\mathrm{N}}$ and $\boldsymbol{H}_{\mathrm{N}}^{*}$.

Let us assume first that these fields are not correlated. Then we have

$$
\left\langle P_{\mathrm{t}}\left(\boldsymbol{r}_{1}\right) P_{\mathrm{t}}\left(\boldsymbol{r}_{2}\right) \cdots P_{\mathrm{t}}\left(\boldsymbol{r}_{j}\right)\right\rangle=\left\langle P_{\mathrm{t}}\left(\boldsymbol{r}_{1}\right)\right\rangle\left\langle P_{\mathrm{t}}\left(\boldsymbol{r}_{2}\right)\right\rangle \cdots\left\langle P_{\mathrm{t}}\left(\boldsymbol{r}_{j}\right)\right\rangle=\left\langle P_{\mathrm{t}}(\boldsymbol{r})\right\rangle^{j} .
$$

Having introduced this relation into the previous formula the developement of the exponential function appears and in the limit $\Delta V \rightarrow 0$ it is

$$
\eta=\exp \left(-n \int \mathrm{d} V\left\langle P_{t}(\boldsymbol{r})\right\rangle\right) \text {. }
$$

The quantum yield is an exponential function of the concentration and of the space integral of the average tunnelling probability, in agreement with Miehlich's observations [6].

This last formula can be calculated exactly only for two special cases. At zero field $\boldsymbol{H}_{0}=\mathbf{0}$, the angle $\psi$ lies directly between $\boldsymbol{H}_{\mathrm{N}}$ and $\boldsymbol{H}_{\mathrm{N}}^{*}$ and the average of $P_{\mathrm{t}}(\boldsymbol{r})$ for an isotropic space distributions of both of them gives

$$
\left\langle P_{\mathrm{t}}\left(\boldsymbol{H}_{0}=0\right)\right\rangle=1-\frac{2 \sqrt{2}}{W}+\frac{4}{W^{2}} \ln (1+W / \sqrt{2}) .
$$

At very high field, above $5 \mathrm{kG}$, the angle $\psi$ tends toward zero and

$$
\begin{aligned}
&\left\langle P_{\mathrm{t}}\left(\left|\boldsymbol{H}_{0}\right| \gg\left|\boldsymbol{H}_{\mathrm{N}}\right|,\left|\boldsymbol{H}_{\mathrm{N}}^{*}\right|\right)\right\rangle \approx P_{2 \mathrm{t}}\left(R, \frac{H_{0}}{T}\right) \approx \\
& \approx \frac{1}{2}\left[\frac{W / \sqrt{2}}{1+W / \sqrt{2}}+\frac{W}{\sqrt{2}} \frac{\pi}{8} \frac{\left\langle H^{\prime}\right\rangle}{H_{0}}+\cdots\right]\left[\cosh \left(\frac{g \beta H_{0}}{2 k T}\right)\right]^{-2} .
\end{aligned}
$$

These two quantities as functions of $W$ are not very different numerically. We can then define a single critical distance $R_{\mathrm{t}}$ for the tunnelling and the corresponding critical volume $V_{t}$ by

$$
V_{\mathrm{t}}=\frac{4}{3} \pi R_{\mathrm{t}}^{3}=\int \mathrm{d} V \frac{W / \sqrt{2}}{1+(W / \sqrt{2})} .
$$


The quantum yield can then be written as

$$
\eta=\exp \left(-n V_{\mathrm{t}}\left\langle P_{\mathrm{t}}\right\rangle\right)
$$

For intermediate values of $\boldsymbol{H}_{0}$ the averaging has to be taken over all pairs of $\boldsymbol{H}_{\mathrm{N}}$ and $\boldsymbol{H}_{\mathrm{N}}^{*}$ and must be computed.

\section{Spin-Lattice Relaxation and EPR}

At temperature higher than $10^{\circ} \mathrm{K}$, the spin-lattice relaxation time in the excited state decreases and mixes up the population of the different pair states within the radiative lifetime [5], changing thereby the average tunnelling probability. The four pair states can be reduced to two species with regard to the tunnelling probability.

If $r$ is the spin-lattice relaxation frequency divided by $w_{\mathbf{r}}$ and

we obtain

$$
W_{1}=W \sin \left(\frac{\psi}{2}\right) / \sqrt{2} \quad W_{2}=W \cos \left(\frac{\psi}{2}\right) / \sqrt{2}
$$

$$
P_{\mathrm{t}}=\frac{W_{1} W_{2}+\left(W_{1}+W_{2}\right)(1 / 2+r)}{W_{1} W_{2}+\left(W_{1}+W_{2}\right)(1+r)+1+2 r} .
$$

At zero applied field, both $W_{1}$ and $W_{2}$ are large within $V_{\mathrm{t}}$ and the relaxation has no effect on $P_{\mathrm{t}}$. At high fields $\left(k T / g \beta \gg H_{0} \gg H_{\mathrm{N}}\right), W_{1}=0$ and

$$
P_{\mathrm{t}}=\frac{1}{2} 1+\frac{W_{2}}{1+W_{2}} \text { for } r \ll 1, \quad P_{\mathrm{t}}=\frac{W_{2}}{W_{2}+2} \text { for } r \gg 1 .
$$

The average tunnelling probability varies then between $1 / 2$ at low temperature (i.e. small $r$ ) and $\mathbf{l}$ at high temperature (i.e. large $r$ ). In this case, the field effect disappears.

If a microwave field of suitable frequency is present, its effect is to enhance the transfer between the spin states and to reinforce the effect of the spin-lattice relaxation. One can then account for it formally by replacing $r$ by $r+m$. This last quantity, normalized with respect to $w_{r}$, involves the microwave power, geometrical factors, etc., as well as the resonant frequency dependence. If the EPR is detected optically by monitoring the luminescence intensity (proportional to the quantum yield $\eta$ ) as a function of the applied field, the signal is defined by

$$
\begin{aligned}
\mathscr{H}_{\text {EPR }} & =\frac{[\eta(m \neq 0)-\eta(m=0)]}{\eta(m=0)}= \\
& =\exp \left\{-n V_{\mathrm{t}}\left[\left\langle P_{\mathrm{t}}(m \neq 0)\right\rangle-\left\langle P_{\mathrm{t}}(m=0)\right\rangle\right]\right\}-1 \leqq 0 .
\end{aligned}
$$

As the EPR is performed at $3 \mathrm{kG}$, we are in the high-field case with $W_{1}=\mathbf{0}$. As only the centres for which $W_{2} \gg 1$ are effective, the change of the tunnelling probability can be simplified to

$$
\Delta P_{\mathrm{t}} \approx \frac{m / 2}{(1+r)(1+r+m)}
$$


The EPR signal obeys then the relation

$$
\ln \left(1-\left|\mathscr{S}_{\mathrm{EPR}}\right|^{-1}\right)=n V_{\mathrm{t}} \frac{1}{2(1+r)} \frac{m}{1+r+m} .
$$

It reveals the resonance in the ground state and in the excited state, because the flip of a single spin suffices to switch a pair into another state. The observed resonances are not sharp because of the unresolved hyperfine splitting. They are Gaussian peaks and their width is directly related to the distribution of the nuclear fields. Assuming for them an isotropic Gaussian distribution of the form

$$
P\left(H_{\mathrm{N} j}\right) \mathrm{d} H_{\mathrm{N} j}=\frac{a}{\sqrt{\pi}} \exp \left(-a^{2} H_{\mathrm{N} j}^{2}\right) \mathrm{d} H_{\mathrm{N} j}, \quad(j=x, y, z)
$$

it can be shown that the field difference $\boldsymbol{H}^{\prime}$ has the same distribution with $a^{\prime-2}=a^{-2}+a^{*-2}$. The average field difference can be determined experimentally from the EPR by

$$
\left\langle H^{\prime}\right\rangle=0.677\left(\Delta H_{1 / 2}^{2}+\Delta H_{1 / 2}^{*^{2}}\right)^{1 / 2},
$$

where $\Delta H_{1 / 2}$ is the full width at half height of the resonance curve. The width of the elementary homogeneous line $T_{2}^{-1}$ is equal to the inverse of the average lifetime before tunnelling, i.e. $W w_{\mathbf{r}}$.

\section{Second-Order Effects}

In the calculation of the luminescent quantum yield, we have assumed that the tunnelling probabilities for different centres are independent, so that the average of the products factorizes. This is true for well-separated centres, but as we require a minimum of proximity to ensure a tunnel effect, we have to examine how a correlation can change the results. The contribution of a single nucleus to the hyperfine field can be expressed by its spin multiplied by a matrix $A$ containing the Fermi contact term and the dipole-dipole interaction:

$$
(A)_{i k}=\gamma \beta_{\mathrm{N}}\left[\frac{8 \pi}{3} \varrho(0) \delta_{i k}+\int \frac{\varrho(r)}{r^{3}}\left(-\delta_{i k}+3 \frac{r_{i} r_{k}}{r^{2}}\right) \mathrm{d}^{3} r\right]
$$

The pairs that we consider contain always a centre with an electron in its ground state. Its spin distribution around each nucleus is spherically symmetric (s-state), so that the interaction integral vanishes identically and the matrix $A$ is reduced to a multiple of the unit matrix $U$. For the excited centre, only the trace of the integral vanishes, because it is not in a pure s-state.

The correlation function for the hyperfine fields on two centres separated by $\boldsymbol{R}$ is proportional to

$$
\left\langle\boldsymbol{H}_{\mathrm{N} 1}(0) \boldsymbol{H}_{\mathrm{N} 2}(\boldsymbol{R})\right\rangle=\sum_{\nu} \sum_{\mu}\left\langle\boldsymbol{I}_{\mu} A_{2 \mu} A_{1 \nu} \boldsymbol{I}_{\nu}\right\rangle
$$

both sums extending over all the surrounding nuclei. As their spins are not correlated $\left(k T \gg \beta_{\mathrm{v}} H\right)$

$$
\left\langle I_{\mu j}\left(A_{2 \mu} \cdot A_{1 \nu}\right)_{j k} I_{\nu k}\right\rangle=\delta_{\mu \nu} \delta_{j k}\left\langle I_{\nu k}^{2}\right\rangle\left(A_{2 v} \cdot A_{1 \nu}\right)_{k k}=\frac{1}{3} I_{v}^{2} \delta_{\mu \nu} \delta_{j k}\left(A_{2 \nu} A_{1 v}\right)_{k k}
$$


and the sum simplifies to

$$
\begin{aligned}
& \left\langle\boldsymbol{H}_{N_{1}}(0) \cdot \boldsymbol{H}_{\mathrm{N} 2}(\boldsymbol{R})\right\rangle=\sum_{\nu} \frac{1}{3} I_{\nu}^{2} \operatorname{Tr}\left(A_{2 v} \cdot A_{1 \nu}\right)= \\
= & \sum_{\nu} \frac{1}{3}\left(\gamma \beta_{N_{v}} I_{v}\right)^{2} \operatorname{Tr}\left\{\frac{8 \pi}{3} \varrho_{1}\left(\boldsymbol{r}_{v}\right) U\left[\frac{8 \pi}{3} \varrho_{2}\left(\boldsymbol{r}_{v}\right) U+\int \cdots \mathrm{d}^{3} r\right]\right\}= \\
= & \sum_{\nu} \frac{1}{3}\left(\gamma \beta_{\mathrm{N} v} I_{v}\right)^{2} \frac{64 \pi^{2}}{3} \varrho_{1}\left(\boldsymbol{r}_{v}\right) \varrho_{2}\left(\boldsymbol{r}_{v}\right) .
\end{aligned}
$$

The spin densities $\varrho_{f}\left(\boldsymbol{r}_{\nu}\right)$ include only the s-component of the wave functions. After replacing the terms $\left(\beta_{\Gamma_{\nu}} I_{\nu}\right)^{2}$ by some adequate average value, the normalized correlation function can be written:

$$
\Gamma(\boldsymbol{R})=\frac{\left\langle\boldsymbol{H}_{\mathrm{N} 1}(0) \cdot \boldsymbol{H}_{\mathrm{N} 2}(\boldsymbol{R})\right\rangle}{\left\langle\boldsymbol{H}_{\mathrm{N} 1}(\boldsymbol{0}) \cdot \boldsymbol{H}_{\mathrm{N} 2}(0)\right\rangle} \approx \frac{\sum_{\nu} \varrho_{\mathrm{s}}^{0}\left(\boldsymbol{r}_{v}\right) \tilde{\varrho}_{\mathrm{s}}^{*}\left(\boldsymbol{r}_{v}+\boldsymbol{R}\right)}{\sum_{v} \varrho_{\mathrm{s}}^{0}\left(\boldsymbol{r}_{v}\right) \tilde{\varrho}_{\mathrm{s}}^{*}\left(\boldsymbol{r}_{v}\right)} \approx \frac{\int \varrho_{\mathrm{s}}^{0}(\boldsymbol{r}) \tilde{\varrho}_{\mathrm{s}}^{*}(\boldsymbol{r}+\boldsymbol{R}) \mathrm{d}^{3} r}{\int \varrho_{\mathrm{s}}^{0}(\boldsymbol{r}) \tilde{\varrho}_{\mathrm{s}}^{*}(\boldsymbol{r}) \mathrm{d}^{3} r} .
$$

The centre wave functions, asymptotically of hydrogenic type, are of the form $\exp \left(r / r_{0}\right)$, and the densities $\exp \left(-2 r / r_{0}\right)$. The tunnelling distance $R_{\mathrm{t}}$ is determined by the overlap of the wave functions and is a certain function of $r_{0}$. The critical correlation distance $R_{\mathrm{e}}$ is therefore almost the same function but of $r_{0} / 2$, so that it should be roughly $R_{\mathrm{t}} / 2$. The volume $V_{\mathrm{c}}$ in which the factorization is not possible is therefore smaller than $V_{t}$, i.e. about $V_{t} / 8$. The correction to be brought to the expression of the quantum yield takes then the form

where

$$
\eta=\mathrm{e}^{-n \boldsymbol{v}_{\mathrm{t}}\left\langle P_{\mathrm{t}}\right\rangle}\left[1+\sum_{v=2}^{\infty} B_{v}+\sum_{v_{\mathrm{g}}>v_{1} \geq 2}^{\infty} \sum_{1}^{\infty} B_{v_{1}} B_{v_{2}}+\sum_{v_{3}>v_{\mathrm{z}}>v_{1} \geqq 2}^{\infty} \sum_{1}^{\infty} B_{v_{1}} B_{v_{2}} B_{v_{3}}+\cdots\right],
$$

$$
B_{v}=\frac{\left(-n V_{\mathrm{c}}\right)^{\nu}}{\nu !} \frac{1}{V_{\mathrm{t}}} \int \mathrm{d} V\left[\left\langle\boldsymbol{P}_{\mathrm{t}}^{\nu}\right\rangle-\left\langle P_{\mathrm{t}}\right\rangle^{\nu}\right]
$$

At high field, the tunnelling probability is quasi-independent of the nuclear configuration, so that the $B^{\prime} \mathrm{s}$ vanish. At zero field, the inside of the tunnelling volume $V_{\mathrm{t}}$ does not contribute to the integrals, because the parameter $W$ is there much larger than unity and the value of the brackets is very small. The integrals are determined only by a thin surface shell $\left(R_{\mathrm{t}}-\delta R_{\mathrm{t}}<R<R_{\mathrm{t}}\right)$, in which $W \approx 1$, and since their value is small, the correction can be limited to the first term

$$
\ln \eta^{-1} \approx n V_{\mathrm{t}}\left\langle P_{\mathrm{t}}\right\rangle\left(1-n V_{\mathrm{c}} K+\cdots\right), K=\frac{1}{2 V_{\mathrm{t}}} \int \mathrm{d} V\left[\left\langle P_{\mathrm{t}}^{2}\right\rangle-\left\langle P_{\mathrm{t}}\right\rangle^{2}\right]
$$

The integrand is of the order of $10^{-2}$, the relative shell thickness is at most of the order of 0.2 (because of the steepness of the function $W(r)$ ) and $V_{\mathrm{c}} \approx V_{\mathrm{t}} / 8$; the correction is not larger than $10^{-3} n V_{\mathrm{t}}$. Since $n V_{\mathrm{t}} \lesssim 1$ for randomly distributed $F$ centres at concentrations of $10^{17} \mathrm{~cm}^{-3}$ this means that correlation effects can be neglected in the practical concentration range.

Another correction has to be introduced when the light absorption is high enough to depopulate appreciably the ground state. If $N$ is the incident flux of photons $\left(\mathrm{cm}^{-2} \mathrm{~s}^{-1}\right), \sigma$ the $\mathrm{F}$ centre cross section for absorption of these pho- 
tons, and $\tau_{c}$ the average time required for a cycle $\mathrm{F}^{0}-\widetilde{\mathbf{F}}^{*}-\mathbf{F}^{0}$, the relative populations $p_{0}$ and $p_{\mathrm{e}}$ in the ground and the excited states are given by

$$
\frac{p_{\mathrm{e}}}{p_{\mathrm{o}}}=N \sigma \tau_{\mathrm{e}}, \quad p_{0}+p_{\mathrm{e}}=1 .
$$

The probability $P_{\mathrm{L}}$ has then to be corrected, because an excited centre has a finite probability that its neighbours are also in the excited state:

$$
P_{\mathrm{L}}=p(0) \cdot 1+p(1)\left[p_{0}\left(1-P_{\mathrm{t}}\right)+p_{\mathrm{e}} 1\right] \approx 1-n \Delta V P_{\mathrm{t}} p_{0} .
$$

The local quantum yield becomes

$$
\eta=\exp \left(-n \int \mathrm{d} V\left\langle P_{\mathbf{t}}\right\rangle p_{0}\right) .
$$

The light absorption being a function of the penetration distance $x$ inside a crystal of thickness $l$, the average quantum yield is given by

$$
\bar{\eta}=\frac{\int_{0}^{l} \mathrm{~d} x \mathrm{e}^{-\sigma n P_{0}(x)} p_{0}(x) \mathrm{e}^{-n V_{t} p_{0}(x)}}{\int_{0}^{l} \mathrm{~d} x \mathrm{e}^{-\sigma n P_{0}(x)} p_{0}(x)},
$$

where

$$
P_{0}(x)=\int_{0}^{x} p_{0}\left(x^{\prime}\right) \mathrm{d} x^{\prime} .
$$

The ground state population is related to the local number of photons and to its derivative by

$$
p_{0}^{-1}-1=\sigma \tau_{\mathrm{c}} N(x), \quad \frac{\partial N}{\partial x}=-N \sigma n p_{\mathrm{o}} .
$$

It is completely determined by the number of photons $N_{0}$ incident on the crystal surface $(x=0)$ and

$$
\frac{N}{N_{0}}+\ln \frac{N}{N_{0}}=1-\frac{x}{X}, \quad X=\frac{N_{0} \tau_{\mathrm{c}}}{n} .
$$

If $N_{0}$ is small, the average quantum yield can be approximated by

$$
\bar{\eta}=\mathrm{e}^{-n V_{\mathrm{t}}\left(1-N_{\mathrm{o}} \sigma \tau_{\mathrm{c}}\right)}, \quad N_{0} \sigma \tau_{\mathrm{c}} \ll \mathbf{l} .
$$

At high flux in a crystal which does not absorb too much, $p_{0}$ is small and nearly constant:

$$
\bar{\eta}=\mathrm{e}^{-n V_{\mathrm{t}} p_{\mathrm{a}}} \approx\left(1-\frac{n V_{\mathrm{t}}}{N_{\mathrm{0}} \sigma \tau_{\mathrm{c}}}\right), \quad N_{0} \sigma \tau_{\mathrm{c}} \gg 1 .
$$

The absorption cross section amounts to some $\AA^{2}$ (e.g. $3 \AA^{2}$ for $\mathrm{KCl}$ ). With a cycle time of $10^{-6} \mathrm{~s}$, the critical flux takes a value around $3 \times 10^{21}$ photons $/ \mathrm{cm}^{2} \mathrm{~s}$, i.e. $1 \mathrm{~kW} / \mathrm{cm}^{2}$. This level can be reached only with a laser, but if the tunnelling is followed by a slow process, the cycle time can be much larger than the radiative lifetime and saturation effects can be expected at a lower illumination power.

Other effects such as $\tilde{\mathrm{F}}^{*}-\tilde{\mathrm{F}}^{*}$ interaction are known to play an important role [9], but are not considered here. 


\section{Comparison with Experiments}

The luminescent intensity has been measured below $10^{\circ} \mathrm{K}$, as a function of a magnetic field between zero and $4 \mathrm{kG}$ in $\mathrm{KBr}, \mathrm{KCl}$, and $\mathrm{KI}$. Table 1 gives the eritical parameters for these substances. The quantum yield is proportional

Table 1

Nuclear magnetic field parameters on $\mathrm{F}$ centres (in $\mathrm{G}$ )

\begin{tabular}{l|c|c|c}
\hline & ground state & $\begin{array}{c}\text { relaxed } \\
\text { excited state } \\
\Delta H_{1 / 2}\end{array}$ & $\left\langle H^{\prime}\right\rangle$ \\
\hline $\mathrm{KCl}$ & $\Delta H_{1 / 2}$ & 79 & 69 \\
$\mathrm{KBr}$ & 65 & 265 & 210 \\
$\mathrm{KI}$ & 148 & 570 & 425
\end{tabular}

to the intensity, but it is obtained only up to a multiplicative constant containing geometrical factors and the excitation intensity. However, its logarithm must be a linear function of the average tunnelling probability $\left\langle\boldsymbol{P}_{\boldsymbol{t}}\right\rangle$. This property allows to determine the parameter $W$ and consequently the unknown average tunnelling frequency, by the following procedure: $\left\langle P_{t}\right\rangle$ is computed with a Monte-Carlo program, in which the nuclear field parameters $a$ and $a^{*}$ (obtained from EPR measurements [5]), the known applied field $\boldsymbol{H}_{0}$ and a guessed value of $W$ are introduced. The logarithm of the luminescent intensity is then plotted as a function of the computed $\left\langle P_{t}\right\rangle$ and a new value of $W$ is chosen

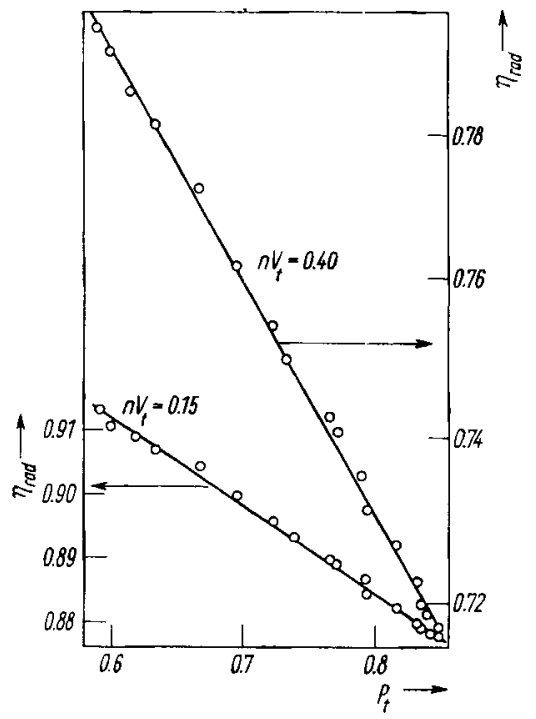

Fig. 2. Experimental radiative quantum yield vs. computed average tunnelling probability in $K I$ at $5.6^{\circ} \mathrm{K}$, for two concentrations. $W$ (average tunnel frequency $x$ radiative lifetime) $=14 \pm 2$

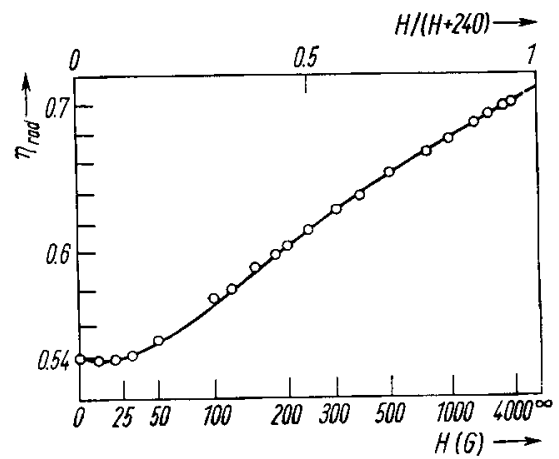

Fig. 3. Radiative quantum yield of $F^{\prime}$ centres vs. magnetic field in $\mathrm{KCl}$ at $6.8^{\circ} \mathrm{K}$. Experimental $(--)$ and computed values (O) at low fields. $\omega_{\mathrm{t}_{0}} / w_{\mathrm{r}}=W=8 \pm 1 ; n V_{\mathrm{t}}=0.80 ; n=3 \times 10^{17} \mathrm{~cm}^{-3} ;$ $R_{\mathrm{t}}=85$ A 
Fig. 4. Measured radiative quantum yield of $F$ centres vs. magnetic field in $\mathrm{KCl}$ at $1.6^{\circ} \mathrm{K}$ (from Porret and Lüty [4])

so as to give the best alignment of the three points corresponding to $\left\langle\boldsymbol{P}_{\mathrm{t}}(0)\right\rangle,\left\langle\boldsymbol{P}_{\mathrm{t}}(\mathbf{4 k G )}\rangle\right.$, and $(1 / 2)\left[\left\langle P_{t}(0)\right\rangle+\left\langle P_{t}(4 k G)\right\rangle\right]$. This is enough to bring all the other points on a straight line, as it can be seen on Fig. 2, which shows the results obtained with $\mathrm{KI}$ at $5.6^{\circ} \mathrm{K}$. The slope yields the value of the product $n V_{t}$, which in turn allows to determine the absolute value of the quantum yield. It has to be pointed out that in this case the concentration $n$ is different from the average concentration measured by optical absorption: the $\mathbf{F}$ centres have been produced by $\mathrm{X}$-rays and their distribution is very inhomogeneous.

The same procedure has been applied to results obtained with $\mathrm{KCl}$ at $6.8^{\circ} \mathrm{K}$, with an homogeneous F-concentration of $3 \times 10^{17} \mathrm{~cm}^{-3}$. The best fit is for $W=8 \pm 1$, with $n V_{\mathrm{t}}=0.80$. The effective radius for tunnelling $R_{\mathrm{t}}$ is therefore about $85 \AA$, in agreement with a theoretical estimate by Lüty and Ferreira [7]. In Fig. 3 we have plotted for the same crystal the calculated and the experimental values of the logarithm of the quantum yield as a function of the applied field. The horizontal scale is not the field itself, but $H_{0} /\left(H_{0}+240 \mathrm{G}\right)$ in order to show more clearly the behaviour at high and at low field. In the former case, the variation of $\log \eta$ is porportional to the inverse field $H_{0}^{-1}$ and in the latter case, both calculation and experiment reveal a slight minimum of the quantum yield at $20 \mathrm{G}$. In $\mathrm{KBr}$ at $6.3^{\circ} \mathrm{K}$, the result is the same, but with $W=11.5 \pm 1$. In all these substances, the average tunnelling probability lies between 10 and $100 \mathrm{~ns}$, in agreement with Ruedin's determination of the homogeneous line width $T_{2}^{-1}$ from EPR saturation.

High-field experiments are also adequately described by this theory. In Fig. 4 we have plotted the logarithm of the inverse of the luminescent quantum yield obtained by Porret and Lüty [4] as a function of $\left[\cosh \left(g \beta H_{0} / 2 k^{\top} T^{\prime}\right)\right]^{-2}$. The experimental points lie very near a straight line passing through the origin, as expected from the formula derived in Section 2 . 'The fit is better if we choose a temperature of $1.6^{\circ} \mathrm{K}$ instead of the $1.7^{\circ} \mathrm{K}$ given by the authors. The fact that the slope is not proportional to the concentration might indicate some clustering of the centres.

\section{Conclusions}

The simple model developed in this paper accounts for the enhancement of the luminescent intensity for all the values of the magnetic field. From optical measurements only, it allows to derive the two main parameters of the tunnelling transfer between neighbouring centres: its average frequency and the effective critical pair separation. The analysis of the temperature behaviour, as performed by Ruedin and co-workers [5] can give information on the spinlattice relaxation by a non-resonant method. Although the large critical pair separation can be explained by the extension of the wave functions, the zero- 
point optical longitudinal vibrations of the lattice must significantly influence the tunnelling process. They produce random electric fields to which the electronic wave functions have plenty of time to adjust. The r.m.s. potential difference between two points at the critical distance can be estimated to lie between 0.1 and $1 \mathrm{~V}$. Since the separation between the conduction band and the $\mathrm{F}$ centre relaxed excited state is at most $0.15 \mathrm{eV}$ (value for $\mathrm{KCl}$ ), the random field should assist the electron transfer. A refined theory should therefore consider also the lattice vibrations and the phonons.

\section{Acknowledgement}

The authors are indebted to the Swiss National Foundation for Scientific Research for the financial support of this work.

\section{References}

[1] R. K. Swank and F. C. Brown, Phys. Rev. 130, 34 (1963).

D. Fröhlich and H. MAHR, Phys. Rev, 140, A656 (1965).

L. Bosi, P. Podran, and G. Spinolo, Phys. Rev. 175, 1133 (1968).

[2] L. Bost, C. Bussotiati, and G. Spinolo, Phys. Rev. B1, 891 (1970).

[3] J. J. Markham, R. T. Platt, and I. L. Mador, Phys. Rev. 92, 597 (1953).

[4] F. Porrex and F. LÜty, Phys. Rev, Letters 26, 843 (1971).

[5] Y. Ruedrn and F. Porret, Helv. phys. Acta 41, 1294 (1968).

Y. Ruedin, P.-A. Schnegg, C. JACCard, and M. Aegerter, in: Magnetic Resonance and Related Phenomena, Ed. I. URsu, Proc. XVIth Colloque Ampère, Bucharest 1970 (p. 892).

Y. RUedin, P.-A. SchnegG, C. JACCard, and M. Aegerter, to be published.

Y. RUEDIN, thesis.

[6] A. Mrehlich, Z. Phys. 176, 168 (1963).

[7] F. LÜTy and G. F. L. Ferreira, private communication.

[8] D. L. Dexter, Phys. Rev. 93, 985 (1954).

[9] K. PARK, Phys. Rev, 140, Al735 (1965).

(Received November 11, 1971) 